\title{
A Study on the Error of a Method for Fiber Orientation Measurement Using Infrared Thermography in Composite Materials
}

\author{
by H. Fernandes*, H. Zhang*, X. Maldague* \\ * Computer Vision and Systems Laboratory (CVSL), Department of Electrical and Computer Engineering, Laval \\ University, 1065 av. De la Médecine, Quebec City, Canada, G1V 0A6. henrique-coelho.fernandes.1@ulaval.ca, \\ xavier.maldague@gel.ulaval.ca
}

\begin{abstract}
Fiber orientation in composite materials is an important feature since the arrangement or orientation of the fibers relative to one another has a significant influence on the strength and other properties of fiber-reinforced composites. In this paper we present a method to assess the fiber orientation on the surface of carbon fiber-reinforced polymer (CFRP) laminates. More specifically, a diode-laser beam is used to locally heat a small spot on the surface of the sample and observation of the heat pattern in the infrared spectrum enables to assess fiber orientation. Different samples and different regions on the surface of the samples are tested in order to estimate the precision of the method.
\end{abstract}

\section{Introduction}

Composite materials are highly used in the industry. One of the reasons is because they have strength and stiffness comparable to metals with the added advantage of significant weight reduction. The fiber orientation, concentration and distribution have a significant influence on the strength and stiffness of fiber-reinforced composites and consequently must follow some quality levels. Thus, one needs to develop testing techniques to assess material's fiber orientation. A destructive approach is not always the best option since the sample would be damage and unfit for use after inspection. In these cases, the use of non-destructive testing (NDT) techniques is required. Infrared thermography (IT) is a widely NDT technique used for diagnostics and motoring in several areas including composite materials. Some of the reasons for its popularity are that it is a safe technique, usually contactless and has a fast inspection rate. In active IT an external heat source is used to stimulate the material being inspected in order to generate a thermal contrast between the feature of interest and the background. The active approach is adopted in many cases given that the inspected parts are usually in equilibrium with the surroundings [1].

In this paper, IT is used in order to assess fiber orientation on the surface of carbon fiber-reinforced polymer (CFRP) laminates. More specifically, a pulse laser heating technique known as Pulsed Thermal Ellipsometry is used to locally spot heat a region on the surface of the sample. If the material has an oriented structure (i.e. fibers orientated in one direction in one layer) with anisotropic thermal properties an elliptical pattern will be observed. The fiber orientation in this case is the same direction of the ellipse major axis. The heating and cowling down profiles are recorded using an infrared camera. The steps required to extract the local fiber orientation from the infrared sequence are presented in this paper. Additionally, tests of several points in different samples were performed in order to estimate the precision of the method.

\section{Pulsed Thermal Ellipsometry - PTE}

More than one century ago, De Senarmont [2] applied a thermal approach to determine the principal orientations in crystal plates: he covered them with a thin layer of wax, heated them over a small spot and monitored the isotherm shape revealed by the solid/liquid transition contour appearing in the wax layer. The isotherm proved to be elliptical and its aspect ratio is related to the square root of the principal conductivities in the surface plane.

This method, referred to later by Krapez et al [5-8] as "Thermal Ellipsometry", was later used for various applications (with, of course, up-to-date experimental equipment) by means of thermography. It was applied on polymer materials to establish a correlation between their draw ratio and the induced thermal anisotropy. It was also used to evaluate the fiber orientation in the case of composite materials using short or long carbon fibers. For the latter problem, authors like Aindow ey al [3] and Cielo et al [4] showed that heat propagates faster in the longitudinal direction of fiber on the surface of fiber reinforced laminates. In [3], Aindow et al. detected local anisotropy in CFRP (nylon-66) injection mouldings by two methods: thermography using infrared scanning, which reveals anisotropy of thermal conductivity, and polarized shear-wave ultrasonic showing elastic anisotropy. For the thermographic method, they recorded isotherms formed around a point source of heat on a plane surface (heat was applied for a period of 15 seconds) using an infrared imaging camera. The isotherms that they observed were ellipses of which the ratio of lengths of the principal axes (b/a) are proportional to the square root of the ratio of thermal conductivities. They assumed that the longest dimension of the counter in each picture indicated the major axis of thermal conductivity in the surface, which in turn is related to the direction of fiber orientation. 


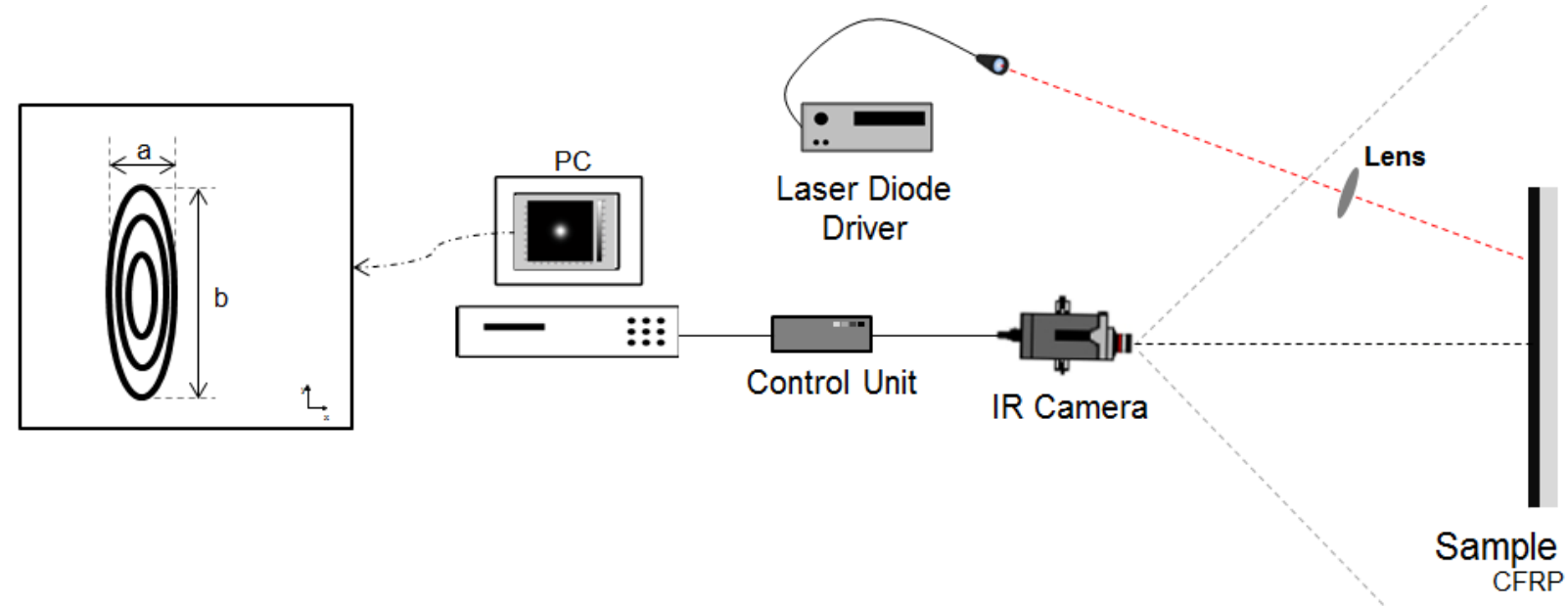

Fig. 1. PTE experimental setup.

Cielo et al. presented in [4] a comparative review of a number of optical techniques for the characterization of non-metallic materials. One possibility reported by them is the evaluation of phase (or fiber) orientation in stretched polymer films or in composites by an analysis of the thermal propagation pattern. They spot-heated the inspected part by a narrow laser beam and the resulting heat-propagation pattern was analyzed by an IR camera. If the material is oriented, such as the unidirectional graphite-epoxy sheet they inspected, an elliptical thermal pattern is observed, with the ratio between the two principal axes (b/a) being related to the square root of the thermal conductivities in the longitudinal and transverse directions. A test on an isotropic material would give a circle instead of an ellipse. They illustrated this approach showing results from two 8-ply unidirectional NARMGO 5217 sheets spot heated for a period of 20 seconds with a $0.5 \mathrm{~W}$ laser.

A more detailed theoretical analysis was later undertaken through an analytical treatment of thermal diffusion in laminates made of orthotropic layers assuming the surface is submitted to concentrated heating by Krapez in [5]. Three temporal regimes were considered in that study: steady-state regime, transient regime (as obtained during step heating), and modulated regime (in order to analyze how the so-called thermal waves "propagate" in orthotropic laminates). Experiments were performed on carbon-epoxy laminates for all three regimes. In [6], Krapez used the same theory (thermal anisotropy measurements method which consists in analyzing the shape of the isotherms which develop around a heated spot) to develop a thermal inversion method to infer thickness of skin and core layers of a 3-layer carbon/epoxy laminate.

In [9], Karpen et al. used lock-in thermography (harmonic thermal waves) to probe orientation fields of carbon fibers both along the surface and in depth at low modulation frequencies and within a short time. Later, in [10] he developed a theoretical model in order to correctly interpret the measurements.

\section{Image segmentation}

Figure 1 show a typical setup used in PTE. After an infrared sequence is recorded it is stored in a 3D matrix M for post-processing. The matrix $M$ is composed of $k$ images of the size $m \times n$. The number of images depends on the acquisition rate of the infrared camera used in the acquisition and the duration of the acquisition. The processing steps in order to obtain the fiber orientation from the acquisition sequence can be divided in three: selection of the optimal diffusion time, binary shape segmentation and extraction of the ellipse's orientation. Figure 2 shows a summary of these steps and they are described next.

\subsection{Optimal diffusion time}

From a PTE inspection we have a sequence of infrared images where the images contain: the plate before heating, the moment when the beam heated the plate, the raise of the temperature profile and finally the temperature profile decrease. In the case of CFRP which are thermally anisotropic, the heat pulse will produce an elliptical pattern on the surface of the sample. The ellipse major axis orientation indicates which direction has the larger thermal conductivity and since the thermal conductivity along the fibers is always larger than the one perpendicular to it the orientation of the major axis of the ellipse will be the same of the fibers.

The thermal pattern is closer to a circle in the images just after the pulse. Shortly after it becomes an ellipse and as time elapses the ellipse become closer to a circle again due to the influence of deeper layers and heat diffusion through thickness and not only on the surface. Thus, in order to assess the fiber orientation on the surface, an image from an early time must be selected when the conductivities of the surface are playing a stronger role. However, the time 
of the selected image must not be too early. If a very early image is selected the heat would not diffused enough on the surface of the plate and consequently the influence of the larger thermal conductivity alongside the fibers will not be strong enough to assess the fiber orientation. Thus, if one extract the thermal pattern shape of each image on the infrared sequence through time could easily observe that the major axis orientation of some of these patterns will be very close to the real fiber orientation and others not.

A fair assumption that could be made is that the pattern that better represents the fiber orientation would be the one which has the larger rate between the ellipse's major axis and its minor axis, i.e. the partner that is 'the most elliptical'. This concept can be abstracted from the definition of the eccentricity of a conic section. Eccentricity (e) is a quantity defined for a conic section which can be given in terms of semimajor and semiminor axes. The eccentricity of a circle is 0 and in the case of an ellipse the eccentricity will be greater than 0 and smaller than 1 . Based on this idea the thermal pattern of each one of the first 60 images (about 1 second) was extracted and its eccentricity calculated. Then, the image with the largest eccentricity value is selected and this image will used for the segmentation of the binary ellipse from which the fiber orientation of the surface will be extract later.



Fig. 2. Image segmentation processing steps

\subsection{Binary shape segmentation}

After the image containing the thermal pattern with the largest eccentricity value is selected, the next step is to segment and binarize the ellipse on the image. This is a simple task that can accomplish by using automatic clustering based image thresholding method. In this work, Otsu's method [11] of image segmentation is used. The algorithm assumes that the image contains two classes of pixels following bi-modal histogram (foreground pixels and background pixels), it then calculates the optimum threshold separating the two classes so that their combined spread (intraclass variance) is minimal. Matlab ${ }^{\circledR}$ native function level = graythresh(img) is available to perform this task where img is the infrared image selected in the previous step and level is the threshold value that is going to be used in the ellipse binarization process. The binary image obeys the following function of belongingness:

$$
\text { fbe }(x, y)= \begin{cases}1, & \text { if } \operatorname{img}(x, y) \geq \text { level } \\ 0, & \text { otherwise }\end{cases}
$$

where $x \in[1,2, \ldots, m], y \in[1,2, \ldots, n]$ and leve/ is the threshold calculated with the Otsu's method.

\subsection{Ellipse orientation extraction}

With the resulting binary image, which is represented by a binary matrix, Matlab ${ }^{\circledR}$ native function regionprops is used to calculate the ellipse orientation. The orientation of the image, here the ellipse, is the angle (in degrees ranging from $-90^{\circ}$ to $90^{\circ}$ ) between the $x$-axis and the major axis of the ellipse that has the same second-moments as the region. Figure 3 illustrates the axes and orientation of the ellipse. The left side of the figure shows an image region and its corresponding ellipse. The right side shows the same ellipse with its major and minor axes. The orientation is the angle between the horizontal dotted line and the major axis. 

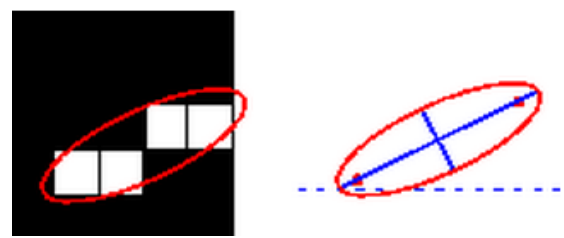

Fig. 3. Small binary image and how its orientation is calculated

\section{Results}

In order to assess the performance of the PTE method, three different CFRP flat laminates were inspected. The first, CFRP001, had 48 plies whose layup was [0/90]24; the second, CFRP002, had 24 plies whose layup was [0/90 $]_{12}$ and the last one, CFRP003, had 24 plies whose layup was $\left[\mathrm{O}_{2} / 90_{2}\right]_{6}$. For all three samples the expected fiber orientation on the surface was $0^{\circ}$. Three different spots were inspected three times each for each sample. Thus, a total of 27 inspections were performed. A waiting time of 3 minutes was respected between each inspection.

The samples were inspected by PTE using a diode-laser spot as stimulation source. The samples were placed, one at a time, in front of the laser beam and a plano-convex lens was placed between the laser beam and the plate (Figure 1). The lens was used to focus the beam in a small spot (about $2 \mathrm{~mm}$ in diameter). The angle of incidence of the laser beam with the plate's surface, i.e. the angle between the incident beam on a surface and the line perpendicular to the surface at the point of incidence, was about $15^{\circ}$ while the camera optical axis angle of incidence was $0^{\circ}$, i.e. the angle between the camera optical axis and the plate's normal to the surface. A short pulse of $0.1 \mathrm{~s}$ was shot heating a small circle area on the plate's surface. Then, the heating and cooling down process were recorded using a mid-wave infrared (MWIR) camera (FLIR Phoenix, InSb, 3-5 $\mu \mathrm{m}$ ). As mentioned before, the pattern formed on the plate's surface is elliptical in anisotropic materials, which is the case of CFRP composite and the ellipse major axis is related to the fiber orientation. The parameters used in this experiment are listed in Table 1.

Table 1. Parameters used during the inspections

\begin{tabular}{|l|l|}
\hline Parameter & Value \\
\hline Diode-laser frequency & $805 \mathrm{mn}$ \\
\hline Diode-laser power & $1 \mathrm{~W}$ \\
\hline Shooting duration & $0.1 \mathrm{~s}$ \\
\hline Spot size on plate's surface & $2 \mathrm{~mm}$ \\
\hline Recording time & $10 \mathrm{~s}$ \\
\hline Camera's acquisition rate & $55 \mathrm{~Hz}$ \\
\hline Camera's frame size & $640 \times 512$ pixels \\
\hline
\end{tabular}

After the samples were inspected and the data stored, the infrared sequences were processed according to what was described in the previous section of this paper. The optimal diffusion time was calculated for each inspection individually. The mean diffusion time was 0.12 seconds. The mean sizes of the binary segmented ellipse's major and minor axis were 11.1 and 8.9 pixels respectively. The orientation of the major axis of each ellipse was then calculated. The mean ellipse major axis orientation was $0.13^{\circ}$ while the standard deviation $2.84^{\circ}$ and the standard error $0.55^{\circ}$. In Table 2 is shown all measured orientations and Figure 4 shows a graphic with all fiber orientations. All orientations are the angle between the $\mathrm{x}$-axis and the major axis of the ellipse.

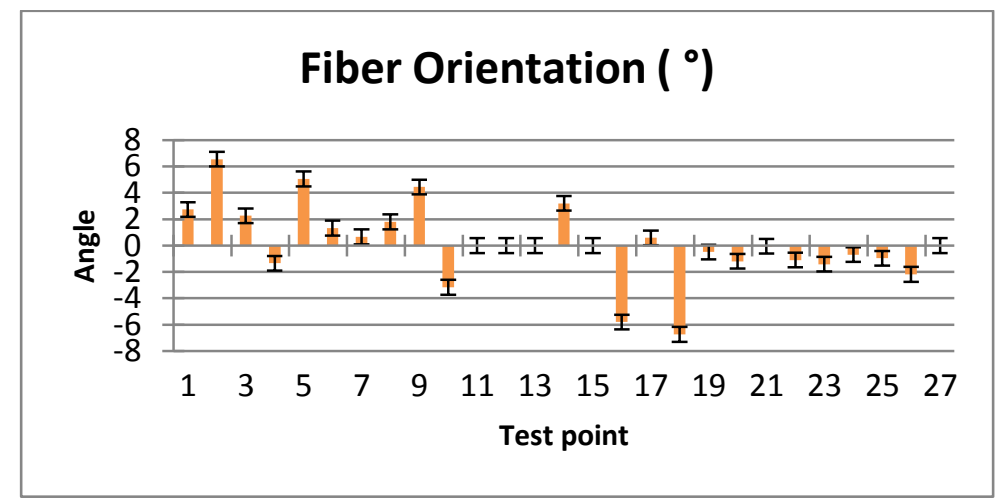

Fig. 4. Fiber orientation of all inspected points with error bars 


\section{Conclusions}

In this work an active infrared thermography technique using diode-laser for fiber orientation measurement on CFRP was presented. A small spot is heated on the surface of the sample and the diffusion of the heat on the surface provides indication of the fiber orientation. In the case of CFRP laminates which have fibers oriented in one direction, the thermal pattern on the surface will have the shape of an ellipse and its major axis orientation will be the same as the direction of the fibers. The selection of the ellipse from the infrared image sequence is done based on the eccentricity of the pattern of each image in the sequence. The image with the largest eccentricity is chosen and from this image, using the Otsu's method for thresholding, a binary image is segmented and fiber orientation of the binary shape calculated.

In order to assess the performance of the described method, three different laminates with known fiber orientation $\left(0^{\circ}\right.$ on the surface) were inspected. Different points on each sample were tested three times each. Results showed that the proposed method can measure the fiber orientation on the surface of a CFRP with an error of $0.55^{\circ}$ which seems to be an acceptable error for an application like this since fiber orientation in CFRP laminates are in most cases typically cut within $\pm 2^{\circ}$ of the nominal targeted fiber orientation angle on each ply.

Table 2. Fiber orientations of inspected points

\begin{tabular}{|c|c|c|c|r|}
\hline & Sample & Point & Inspec.\# & Orient. \\
\hline 1 & CFRP001 & A1 & 1 & $2.73^{\circ}$ \\
\hline 2 & CFRP001 & A1 & 2 & $6.54^{\circ}$ \\
\hline 3 & CFRP001 & A1 & 3 & $2.25^{\circ}$ \\
\hline 4 & CFRP001 & B1 & 1 & $-1.34^{\circ}$ \\
\hline 5 & CFRP001 & B1 & 2 & $5.05^{\circ}$ \\
\hline 6 & CFRP001 & B1 & 3 & $1.32^{\circ}$ \\
\hline 7 & CFRP001 & C1 & 1 & $0.66^{\circ}$ \\
\hline 8 & CFRP001 & C1 & 2 & $1.79^{\circ}$ \\
\hline 9 & CFRP001 & C1 & 3 & $4.44^{\circ}$ \\
\hline \hline 10 & CFRP002 & A2 & 1 & $-3.16^{\circ}$ \\
\hline 11 & CFRP002 & A2 & 2 & $0^{\circ}$ \\
\hline 12 & CFRP002 & A2 & 3 & $0^{\circ}$ \\
\hline 13 & CFRP002 & B2 & 1 & $0^{\circ}$ \\
\hline 14 & CFRP002 & B2 & 2 & $3.2^{\circ}$ \\
\hline 15 & CFRP002 & B2 & 3 & $0^{\circ}$ \\
\hline 16 & CFRP002 & C2 & 1 & $-5.79^{\circ}$ \\
\hline 17 & CFRP002 & C2 & 2 & $0.58^{\circ}$ \\
\hline 18 & CFRP002 & C2 & 3 & $-6.73^{\circ}$ \\
\hline \hline 19 & CFRP003 & A3 & 1 & $-0.48^{\circ}$ \\
\hline 20 & CFRP003 & A3 & 2 & $-1.19^{\circ}$ \\
\hline 21 & CFRP003 & A3 & 3 & $-0.05^{\circ}$ \\
\hline 22 & CFRP003 & B3 & 1 & $-1.09^{\circ}$ \\
\hline 23 & CFRP003 & B3 & 2 & $-1.41^{\circ}$ \\
\hline 24 & CFRP003 & B3 & 3 & $-0.69^{\circ}$ \\
\hline 25 & CFRP003 & C3 & 1 & $-0.96^{\circ}$ \\
\hline 26 & CFRP003 & C3 & 2 & $-2.17^{\circ}$ \\
\hline 27 & CFRP003 & C3 & 3 & $0^{\circ}$ \\
\hline
\end{tabular}




\section{ACKNOWLEDGMENTS}

The authors would like to gratefully acknowledge support provided by the Canada Research Chair in Multipolar Infrared Vision (MiViM) and the industrial partners: Bell Helicopter Textron Canada Limited, Bombardier Inc., Pratt and Whitney Canada Corp., Avior Integrated Products Inc., Delastek Inc. and Hutchinson Inc. Canada. The authors would also like to acknowledge the support of the following agencies: CNPq, National Council for Scientific and Technological Development - Brazil; NSERC, Natural Sciences and Engineering Research Council of Canada; FQRNT, Quebec Fund for Research on Nature and Technology and CRIAQ, Consortium for Research and Innovation in Aerospace in Quebec.

\section{REFERENCES}

[1] Maldague X., "Theory and practice of infrared technology for nondestructive testing". John Wiley \& Sons, New York, 2001.

[2] De Senarmont M. H., "Mémoire sur la conductibilité des substances cristallisées pour la chaleur". Second Mémoire, Annales de Chimie Physique, vol. 3, 22, pp. 179-211, 1851.

[3] Aindow J.D., Markhan M.F., Puttick K.E., Rider J.G. and Rudman M.R., "Fibre orientation detection in injectionmoulded carbon fibre reinforced components by thermography and ultrasonics". NDT international, vol. 19 , vo. 1, pp. 24-29, 1986.

[4] Cielo P., Maldague X., Krapez J.-C. and Lewak R., "Optics-Based Techniques for the Characterization of Composites and Ceramics". Nondestructive Characterization of Metals; Montreal; Canada, pp 733-744, 1987.

[5] Krapez J.-C., "Thermal ellipsometry applied to the evaluation of fibre orientation in composites". Journee d'Etude sur la Thermographie, Paris, France, no. 1994-171, 1994.

[6] Krapez J.-C., "Thermal ellipsometry - A tool applied for in-depth resolved characterization of fibre orientation in composites". Review of Progress in Quantitative Nondestructive Evaluation, Seattle, WA, July 30-Aug. 4, 1995, ONERA, TP, no.1995-148, 1995.

[7] Krapez J.-C., Gardette G. and Balageas D., "Thermal ellipsometry in steady-state and by lock-in thermography. Application to anisotropic materials characterization". In: D. Balageas, G. Busse, G.M. Carlomagno, editors. Proceedings of the Qirt 96, Eurotherm Series 50. EETI ed., pp. 257-262, 1996.

[8] Krapez J.-C. and Gardette G., "Characterisation of anisotropic materials by steady-state and modulated thermal ellipsometry”. High Temperatures - High Pressures, vol. 30, no. 5, pp. 567-574, 1998.

[9] Karpen W., Wu D., Steegmuller R. and Busse G., "Depth profiling of orientation in laminates with local lock-in thermography", in: D. Balageas, G. Busse, G.M. Carlomagno, editors. Proceedings of the Qirt 94, Eurotherm Series 42, EETI editions, pp. 281-286, 1994.

[10] Karpen W., Wu D. and Busse G., "A Theoretical Model for the Measurement of Fiber Orientation with Thermal Waves". Res. Nondestr. Eval., vol. 11, pp. 179-197, 1999.

[11] Otsu N., "A threshold selection method from gray-level histogram". IEEE Trans. Systems Man Cybern, vol. 9, no. 1, pp. 62-66, 1979. 